\title{
Síndrome serotoninérgico. Presentación de cuatro casos y revisión de la literatura
}

\author{
P. YOUNG, B. C. FINN, F. ÁLVAREZ, M. F. VERDAGUER', F. J. BOTTARO, \\ J. E. BRUETMAN \\ Servicio de Clínica Médica y ${ }^{\prime}$ Psiquiatría. Hospital Británico de Buenos Aires. Argentina
}

\author{
SEROTONIN SYNDROME: FOUR CASES REPORT AND REVIEW OF \\ THE LITERATURE
}

\begin{abstract}
RESUMEN
El síndrome serotoninérgico es una condición clínica asociada al uso de medicamentos que promueven la neurotransmisión serotoninérgica. El conjunto de sus manifestaciones, mentales, autonómicas y neuromusculares, resulta de una sobre-estimulación de receptores de serotonina, centrales y periféricos. Usualmente, el cuadro resuelve dentro de las primeras 24 horas, de suspensión del fármaco vinculado a su producción. Sin embargo, algunos de los pacientes progresan a fallo multiorgánico y mueren.

Presentamos cuatro pacientes añosos, que se presentaron con la tríada clásica, en promedio, al tercer día desde el inicio o una variación del tratamiento con inhibidores de la recaptación de serotonina. Todos respondieron favorablemente con la suspensión del fármaco y, en tres de los cuatro casos, con el agregado de clorpromazina. Creemos que es un cuadro subdiagnosticado, potencialmente fatal, pero reversible si se toma tiempo, cuya identificación oportuna exige un alto índice de sospecha.
\end{abstract}

\begin{abstract}
The serotonin syndrome is a clinical condition associated with serotonin agonists. Is due to an overstimulation of central and peripheral serotonin receptors that leads to mental, autonomic and neuromuscular changes. Usually the disorder resolves within the first 24 hours after the medications are discontinued, however some patients progress to a multiple organ failure and die.

We describe four elderly patients that presented with the classic triad. They presented the symptoms in average at the third day after the initiation or variation of the treatment with serotonin reuptake inhibitors. All had a favorable response with the suspension of medications and, in three cases, with the treatment with chlorpromazine.

We believe it is a potentially fatal but reversible condition, probably underdiagnosed that requires a high index of suspicion.
\end{abstract}

KEY WORDS: Serotonin syndrome. Serotonin. Diagnosis. Treatment.

PALABRAS CLAVE: Síndrome serotoninérgico. Serotonina. Diagnóstico. Tratamiento.

Young P, Finn BC, Álvarez F, Verdaguer MF, Bottaro FJ, Bruetman JE. Síndrome serotoninérgico. Presentación de cuatro casos y revisión de la literatura. An Med Interna (Madrid) 2008; 25: 125-130.

\section{INTRODUCCIÓN}

El síndrome serotoninérgico (SS) representa un conjunto de signos y síntomas atribuidos a una actividad excesiva de la neurotransmisión serotoninérgica en el sistema nervioso central y periférico (1). La mayoría de los casos reportados se asocia al consumo de medicamentos que, por distintos mecanismos, multiplican la estimulación de receptores de serotonina en células nerviosas $(1,2)$. Con el aumento del uso de los inhibidores selectivos de la recaptación de serotonina (ISRS) desde 1990, asociado a otros medicamentos, la incidencia y el reconocimiento de éste síndrome va en aumento (2). Se caracteriza por una tríada clínica de alteración del status mental, trastornos disautonómicos y alteraciones neuromusculares, de presentación variable y no siempre completa (1-3). La gravedad del cuadro es también variable, y la mayoría de los casos alcanzan la remisión completa con la suspensión de la droga asociada a medidas de soporte. Es un cuadro potencialmente fatal, pero reversible (4). Se describen cuatro casos internados en el Hospital Británico entre los años 2004-2006, y una revisión de la literatura.

\section{CASOS APORTADOS}

Caso 1. Paciente de sexo femenino y 77 años con antecedentes de hipertensión arterial (HTA), fibrilación auricular anticoagulada, enfermedad del nodo con requerimiento de marcapasos unicameral, insuficiencia cardíaca diastólica, gammapatía monoclonal de significado incierto con diagnóstico en el 2003 y en control anual. En tratamiento con enalapril $5 \mathrm{mg} /$ día (mg/día), atenolol $25 \mathrm{mg} /$ día, y ácido acetilsalicílico $200 \mathrm{mg}$ /día. Dos meses antes de la presentación del cuadro, inició tratamiento con fluoxetina, en dosis crecientes hasta $40 \mathrm{mg}$ /día, y bromazepan $3 \mathrm{mg} /$ día por síndrome depresivo. Cuatro días antes de la internación consultó a un reumatólogo, quien le indicó un preparado con propoxifeno $80 \mathrm{mg}$, glucosan $300 \mathrm{mg}$, meloxicam $15 \mathrm{mg}$, omeprazol $20 \mathrm{mg}$ y betametasona $0,4 \mathrm{mg}$. Ingresó a cui- 
dados intensivos con un cuadro de letargia, Glasgow 11/15 (respuesta verbal confusa y apertura ocular al estímulo doloroso, localiza al dolor), sudoración profusa, mioclonías e hiperreflexia, con midriasis, y sin signos neurológicos focales. Al ingreso se presentó afebril, con frecuencia cardíaca de 110 latidos por minuto, presión arterial (PA) de $180 / 90 \mathrm{~mm} \mathrm{Hg}$, frecuencia respiratoria de 20 por minuto, $\mathrm{SaO} 296$ $\%$ al aire ambiente. Durante las primeras 24 horas de internación presentó un cuadro de agitación severa, que fue contralado con benzodiacepinas. El laboratorio no evidenció ninguna anormalidad (hemograma, hepatograma, glucemia, coagulograma creatininemia, uremia, ionograma con magnesio, calcio, fósforo, creatinfosfoquinasa, velocidad de sedimentación globular, función tiroidea, gases arteriales y sedimento urinario). El ECG y la Rx de tórax fueron normales. Se realizó una tomografía axial computada de cerebro (TAC) que no mostró lesiones. El electroencefalograma (EEG) demostró actividad lenta, continua y generalizada, compatible con encefalopatía metabólica. Se realizó una punción lumbar con citoquímico normal y cultivo negativo, así como dos hemocultivos y un urocultivo que fueron negativos. Se interpretó el cuadro como SS, provocado por la superposición de fluoxetina con omeprazol. Se suspendió la fluoxetina, y se inició tratamiento con clorpromazina $25 \mathrm{mg}$ cada 12 horas por vía oral con evolución favorable hasta el egreso hospitalario al séptimo día de internación. En control por consultorios a las dos semanas, se suspendió la clorpromazina.

Caso 2. Paciente de sexo femenino y 90 años, con antecedentes de HTA y síndrome depresivo. En tratamiento con enalapril 10 $\mathrm{mg}$ /día y mianserina $30 \mathrm{mg}$ /día. En los últimos 7 días presentó agravamiento del cuadro depresivo, en el contexto de la muerte reciente de una hija, e inició tratamiento con escitalopram 20 mg/día.

Fue traída por familiares al presentar un cuadro de desorientación témporo-espacial, de instalación brusca, y asociado a registros de HTA de 190/100 mm Hg, nauseas, vómitos y diarrea. Ingresó a sala general, donde se constataba confusión marcada, agitación, alucinaciones visuales y temblor fino en ambas manos, con clonus espontáneo e hiperreflexia con predominio en miembros inferiores, sin foco neurológico. Presentó frecuencia cardíaca de 120 latidos por minuto, PA de 190/100 $\mathrm{mm} \mathrm{Hg}$, frecuencia respiratoria 20 por minuto, temperatura axilar $38,90{ }^{\circ} \mathrm{C}, \mathrm{SaO}_{2} 95 \%$ al aire ambiente. El laboratorio no puso de manifiesto ninguna anormalidad. El ECG y la Rx de tórax fueron normales. Se realizó una TAC de cerebro que no mostró lesiones. El EEG se encontró dentro de límites normales. Se realizó una punción lumbar con citoquímico normal y cultivo negativo, 2 hemocultivos y un urocultivo, que también fueron negativos. A las 72 horas continuaba confusa e hipertérmica. Se solicitó la interconsulta con psiquiatría. En el interrogatorio, la persona encargada de su cuidado habitual informaba el uso irregular no prescripto de dosis diarias excesivas de escitalopram, de acuerdo con el estado subjetivo inmediato; y con esta información se formuló la hipótesis diagnóstica de SS. Se Inició nitroglicerina en goteo por registros de HTA de 210/120 mm Hg sin control satisfactorio. Con el diagnóstico, se suspendió el escitalopram y se inició tratamiento con clorpromazina $25 \mathrm{mg}$ cada 12 horas por vía oral, con evolución favorable, normalización del sensorio y los parámetros vitales, siendo posible el egreso hospitalario al décimo día de internación. En control por consultorios se suspendió la clorpromazina.

Caso 3. Paciente de sexo femenino de 79 años con antecedentes depresión mayor crónica de cualidades melancólicas, intento de suicidio en el año 2003, y deterioro cognitivo leve. En tratamiento prolongado con sertralina $100 \mathrm{mg}$ /día, ácido valproico $500 \mathrm{mg}$ /día y clonazepan $2 \mathrm{mg} /$ día; había presentado en el último año diarrea acuosa crónica. En el curso de la misma, la paciente se negó a realizar estudios endoscópicos, el coproparasitológico fue negativo, las hormonas tiroideas normales, y la serología para enfermedad celíaca negativa. Ingresó a comienzos del año 2006 a sala general, por confusión, hipertermia, y empeoramiento del cuadro de diarrea con diez deposiciones al día. Esta sintomatología coincidió con el mal manejo de la medicación en domicilio, ya que la paciente habría ingerido un total aproximado de $400 \mathrm{mg}$ de sertralina en las últimas 24 horas. A su ingreso presentaba frecuencia cardíaca de 104 latidos por minuto, PA de $140 / 100 \mathrm{mmHg}$, frecuencia respiratoria de 24 por minuto, temperatura axilar de $380{ }^{\circ} \mathrm{C}, \mathrm{SaO}_{2} 98 \%$ al aire ambiente. Con respuesta parcial a órdenes simples, confusa, con clonus inducible e hiperreflexia osteotendinosa con predominio en miembros inferiores, mioclonías con predominio en miembros superiores, temblores generalizados, y sin signos neurológicos focales. Se constató sudoración profusa y diarrea. El laboratorio no evidenció ninguna anormalidad. El ECG y la Rx de tórax eran normales. Se realizó una TAC de cerebro que no mostró lesiones agudas, y resonancia magnética nuclear con angioresonancia de polígono y vasos de cuello, difusión y cortes para visualización del lóbulo temporal, resultaron dentro de los límites normales para su edad. El EEG demostró lentificación difusa de la actividad de fondo. Se realizó una punción lumbar con citoquímico normal, cultivo negativo, reacción de la polimerasa para herpes simplex tipo 1 y 2 negativos, 2 hemocultivos, urocultivo y coprocultivo que fueron negativos. Dado el incremento abrupto de la dosis de sertralina, en el contexto de un tratamiento que la combinaba con ácido valproico, se planteó el diagnóstico de SS, se suspendió el antidepresivo, se roto el ácido valproico a lamotrigina y se inició la administración de clorpromazina $25 \mathrm{mg}$. El clonazepan no se suspendió durante la internación. La paciente recibió ceftriaxona empírica hasta el cuarto día en que se confirmaron los cultivos negativos. La fiebre desapareció al sexto día de internación. Con evolución favorable, egresó a las dos semanas. En control por consultorios externos, a los siete días del egreso se suspendió la clorpromazina.

Caso 4. Paciente de sexo masculino y 68 años, con antecedentes de síndrome depresivo en tratamiento desde enero de 2004 con fluoxetina $20 \mathrm{mg} /$ día, a la que se agregó, en agosto del mismo año, amitriptilina en dosis crecientes hasta llegar a $75 \mathrm{mg} /$ día. Dos meses más tarde se aumentó la dosis de amitriptilina a $150 \mathrm{mg} /$ día. Ingresó a las $24 \mathrm{hs}$ del incremento de la amitriptilina por presentar somnolencia, conducta agresiva, hipertermia, diarrea y acatisia marcada. A su ingreso se presentaba con frecuencia cardíaca de 110 latidos por minuto, PA de $160 / 90 \mathrm{mmHg}$, frecuencia respiratoria de 20 por minuto, temperatura axilar de $390{ }^{\circ} \mathrm{C}, \mathrm{SaO}_{2} 98 \%$ al aire ambiente. Con respuesta parcial a órdenes simples, somnoliento, con clonus espontáneo predominante en miembros inferiores, mioclonías en cuatro miembros, temblores generalizados, y sin signos neurológicos focales. Se constató sudoración profusa y diarrea. El laboratorio de ingreso mostró Hto de 35\%, Hb de 11,7 g/dl, leucocitos de 10.200 $\mathrm{mm}^{3}$, glucemia de $111 \mathrm{mg} / \mathrm{dl}$, uremia de $48 \mathrm{mg} / \mathrm{dl}$, creatininemia de $0,98 \mathrm{mg} / \mathrm{dl}$, eritrosedimentación de $50 \mathrm{~mm} /$ hora, sodio de $138 \mathrm{mEq} / \mathrm{l}$, potasio de $3,7 \mathrm{mEq} / 1$, TGO $96 \mathrm{U} / 1$, TGP $102 \mathrm{U} / 1$, fosfatasa alcalina de $120 \mathrm{U} / 1$, resto dentro de valores normales. El ECG y la Rx de tórax eran normales. Se realizó una TAC de cerebro que no mostró lesiones agudas y resonancia magnética nuclear de cerebro con difusión dentro de los límites normales para su edad. El EEG demostró lentificación difusa de la actividad de fondo. Se realizó una punción lumbar con citoquímico normal y cultivo negativo, dos hemocultivos y un urocultivo que fueron negativos. Dado el incremento de la dosis de amitriptilina y su sintomatología, se propuso el diagnóstico de SS, se suspendieron la fluoxetina y la amitriptilina, y se instauraron medidas de sostén con buena evolución. Egresó del hospital a los 10 días de internación.

\section{DISCUSIÓN}

El SS fue descripto por primera vez en 1960 por Oates y cols. (5) en siete pacientes depresivos tratados con inhibidores de la monoaminooxidasa (IMAO) y L-triptofano. Sin embargo, el nombre fue utilizado inicialmente por Insel y cols., en 1982 (6). En 1991 Sternbach, publicó el primer artículo de revisión recopilando un total de 38 casos (7); y, hasta la fecha, han sido descriptos aproximadamente 300 casos (1-8). La 
incidencia del SS se encuentra en aumento, y esto se explica por el uso generalizado de los ISRS y otros fármacos que aumentan el tono serotoninérgico. Hasta 2002 se habían reportado 7.349 casos de toxicidad y 93 muertes asociadas con los efectos tóxicos de los ISRS entre un grupo de 26.733 pacientes tratados (9). Entre los pacientes ancianos, la mortalidad fue mayor al 11\%. El SS ocurre aproximadamente en el 14 a $16 \%$ de los pacientes con sobredosis de ISRS (9).

La serotonina es el neurotransmisor de un sistema de proyección difusa originado en células del rafe en el tronco encefálico. Juega un rol muy importante en el ciclo sueño-vigilia, en el estado de ánimo, en las conductas relacionadas con la emoción, en la alimentación, y en la termorregulación; y, a nivel periférico, interviene en la regulación del tono vascular, y la motilidad gastrointestinal, entre otras funciones (4).

El SS es el resultado de la sobre-estimulación de receptores de 5-hidroxi-triptamina $1 \mathrm{~A}\left(5-\mathrm{HT}_{1 \mathrm{~A}}\right)$ en núcleos grises centrales y del bulbo, y de la sobre-estimulación de receptores 5$\mathrm{HT}_{2 \mathrm{~A}}(1,2)$. No es una reacción idiosincrática, sino que representa una consecuencia predecible del estímulo disfuncional (2). Habitualmente se presenta en forma repentina, dentro de las primeras 24 horas de iniciada la administración de un agente serotoninérgico, la modificación de la dosis, o la suma de un nuevo fármaco a un tratamiento establecido (1-4). La actividad excesiva de los receptores puede resultar de un exceso de precursores de serotonina, el aumento de su liberación, la disminución de la recaptación, la disminución del metabolismo de inactivación, o la sobre-estimulación postsináptica de los receptores (1-4). En la tabla I se nombran las diferentes drogas que, por múltiples mecanismos, son capaces de dar lugar a un SS. Otros fármacos que podrían producir el síndrome son: mianserina, ondansetrón, y metoclopramida (2).

Los pacientes ancianos representan un subgrupo vulnerable. Su ocurrencia se asocia con la polifarmacia, a menudo con la utilización de dos o más medicamentos $(1,2)$. En una serie, la edad promedio de aparición del SS fue 40 años (3).

Cabe destacar que una sola dosis de un ISRS puede producir el SS (1-4). Las drogas que inhiben las enzimas del citocromo P450, que metabolizan los agentes serotoninérgicos, también aumentan la incidencia del síndrome (4). Entre estas drogas se cuentan la eritromicina, la claritromicina, el ketoconazol y el omeprazol, entre otros $(4,10,11)$. Existen dos rutas de metabolismo para la fluoxetina por el citocromo P450 (CPY): la $N$-demetilación a norfluoxetina por CPY2C19 y CYP2C9, y en menor medida la CPY3A4; y la $O$-dealquilación a trifluorometilfenol por CPY2C19 Y CPY3A4. El omeprazol es un inhibidor de la CPY2C19, capaz de aumentar los niveles plasmáticos de fluoxetina en 120 veces, incrementando su toxicidad (11).

La frecuencia del fenotipo de metabolizadores lentos para CPY2C19 en caucásicos es del 2 al 5\%, y en orientales es de hasta el $20 \%(11,12)$. Se ha demostrado que un $7 \%$ de la población está compuesto por metabolizadores lentos para los ISRS (4). La administración de un agente serotoninérgico dentro de las cinco semanas de suspensión del tratamiento con fluoxetina podría producir un SS, habida cuenta de las vidas medias prolongadas de la fluoxetina y su metabolito activo, norfluoxetina $(1,2)$. La utilización de IMAO, se asocia a casos

\section{TABLA I}

MECANISMOS DE ACCIÓN DE DROGAS QUE SE ASOCIAN A SÍNDROME SEROTONINÉRGICO

\begin{tabular}{|c|c|c|c|c|}
\hline Sintesis de 5-HT & Liberación de 5-HT & $\begin{array}{l}\text { Inhibición de } \\
\text { recaptación 5-HT }\end{array}$ & $\begin{array}{l}\text { Inhibición del } \\
\text { metabolismo de 5-HT }\end{array}$ & $\begin{array}{l}\text { Estimulación receptor } \\
\text { post-sináptico }\end{array}$ \\
\hline $\begin{array}{l}\text { L-triptófano } \\
\text { 5-hidroxitriptófano }\end{array}$ & $\begin{array}{l}\text { Anfetaminas y derivados } \\
\text { Dextroanfetamina } \\
\text { Metanfetamina } \\
\text { Fenfluramina } \\
\text { Dexfenfluramina } \\
\text { Fenteramina } \\
\text { Éxtasis } \\
\text { Cocaína } \\
\text { Reserpina } \\
\text { Tetrabenazina } \\
\text { Levodopa } \\
\text { IMAO } \\
\text { Fenelzina } \\
\text { Tranilcipromina } \\
\text { Seleginina } \\
\text { Moclobemida } \\
\text { Isocarboxazida }\end{array}$ & $\begin{array}{l}\text { ISRS } \\
\text { Fluoxetina } \\
\text { Paroxetina } \\
\text { Sertralina } \\
\text { Fluvoxamina } \\
\text { Citalopram } \\
\text { Trazodona } \\
\text { Nefazodona } \\
\text { Venlafaxina } \\
\text { ADT } \\
\text { Amitriptilina } \\
\text { Imipramina } \\
\text { Clomipramina } \\
\text { Doxepina } \\
\text { Desipramina } \\
\text { Bupropión } \\
\text { Dextrometorfano } \\
\text { Tramadol } \\
\text { Meperidina } \\
\text { Sibutramina } \\
\text { Cocaína } \\
\text { Hipérico } \\
\text { Anfetaminas y deriv }\end{array}$ & $\begin{array}{l}\text { IMAO: } \\
\text { Fenelzina } \\
\text { Tranilcipromina } \\
\text { Seleginina } \\
\text { Moclobemida } \\
\text { Hipérico } \\
\text { Omeprazol } \\
\text { Acido Valproico } \\
\text { Ketoconazol } \\
\text { Linezolid } \\
\text { Ritonavir }\end{array}$ & $\begin{array}{l}\text { Buspirona } \\
\text { Agonistas 5-HT1 } \\
\text { Sumatriptán } \\
\text { Zolmitriptán } \\
\text { Naratriptán } \\
\text { Rizatriptán } \\
\text { Mirtazapina } \\
\text { Litio } \\
\text { Carbamazepina }\end{array}$ \\
\hline
\end{tabular}

IMAO: inhibidores de la recaptación de serotonina; ADT: antidepresivos tricíclicos; ISRS: inhibidores de la recaptación de serotonina; 5-HT 1: 5-hidroxi triptamina 1. 
severos especialmente cuando se los utiliza en combinación con meperidina, dextrometorfano, ISRS, o éxtasis (2). Se han reportado también otras combinaciones de drogas capaces de producir un SS severo (2).

El SS puede presentarse con un espectro amplio de signos y síntomas, y existen numerosas barreras que limitan al clínico para hacer el diagnóstico de esta condición. Primero, este cuadro puede tener características proteiformes, y el médico puede malinterpretar síntomas, como temblor, diarrea o hipertensión, y no relacionarlos al tratamiento. En segundo lugar, la aplicación estricta de los criterios de Sternbach modificados deja sin diagnóstico lo que ahora se conoce como formas leves, tempranas, y aún las subagudas. Por último, los médicos no diagnostican esta condición por el desconocimiento de un cuadro que ya no es raro y se presenta en todas las edades (1-4).

No existe un test específico para el diagnóstico del SS, que es clínico, y debe apoyarse en la historia de uso de un agente serotoninérgico, un espectro variable de signos y síntomas, que van desde temblor y diarrea en los casos leves, hasta rigidez neuromuscular e hipertermia en los casos graves.

Radomski y cols. (12). revisaron los criterios diagnósticos descriptos por Sternbach (7), y los definieron de la siguiente manera:

- Aumento de dosis o adición reciente de un agente serotoninérgico a un tratamiento ya establecido, y la presencia de 4 síntomas mayores o 3 síntomas mayores y dos menores (Tabla II).

- Estos síntomas no deben corresponder a un trastorno psiquiátrico (o su intensificación), que estaba presente antes de que el paciente recibiera el agente serotoninérgico.

-Exclusión de causas infecciosas, metabólicas, endócrinas o tóxicas.

- No debe haber sido introducido un tratamiento antipsicótico, ni aumentadas sus dosis antes de la presentación de los síntomas.

En el año 2003 Dunkley y cols., (8). propusieron nuevos criterios diagnósticos que, comparados con los criterios originales, son más simples de utilizar, más sensibles (84 vs. 75\%), y específicos (97 vs. 96\%). Estos describen, en primera instancia, el uso de un agonista serotoninérgico en las 5 semanas previas al evento, y la presencia de alguno de los siguientes

TABLA II

SÍNTOMAS MAYORES Y MENORES

\begin{tabular}{lcc}
\hline Alteración & Síntomas mayores & Síntomas menores \\
\hline Status mental & Confusión & Hiperactividad \\
& Coma & Agitación \\
& Insomnio \\
Autonómica & Hipertermia & Taquicardia \\
& Diaforesis (sudoración) & Taquipnea \\
& & Disnea \\
Neuromuscular & Hipo/Hipertensión \\
& Mioclonus e hipertonía & Diarrea \\
& (espontáneo, inducible, ocular) & Incoordinación \\
& Hiperreflexia & Midriasis \\
& Temblor & Akatisia \\
& & Ataxia \\
\hline
\end{tabular}

grupos de síntomas: a) temblor o hiperreflexia; b) clonus espontáneo; c) rigidez muscular, temperatura $>38{ }^{\circ} \mathrm{C}$, y clonus ocular o inducible; d) clonus ocular, agitación y/o sudoración; y e) clonus inducible, agitación y/o sudoración (8). El clonus ocular es un movimiento ocular horizontal rítmico de amplio rango, también llamado ojos en ping-pong.

Los hallazgos clínicos con una asociación estadísticamente significativa con el SS fueron en primera medida los neuromusculares, que incluyen el clonus inducible, espontáneo y ocular. Éstas son las manifestaciones más importantes para establecer el diagnóstico $(2,8)$. El clínico debe estar alertado de que la rigidez puede enmascarar la hiperreflexia y el clonus (2).

En los casos leves, el paciente puede estar afebril pero taquicárdico; con hallazgos autonómicos al examen físico como escalofríos, sudoración y midriasis; y el examen neurológico puede revelar temblor intermitente, mioclonus, hiperreflexia. Los casos moderados se caracterizan por alteración de los signos vitales con taquicardia, hipertensión e hipertermia. La temperatura puede ser mayor a $40^{\circ} \mathrm{C}$. $\mathrm{Al}$ examen físico se puede observar midriasis, ruidos hidroaéreos aumentados, sudoración con coloración normal de la piel. La hiperreflexia y clonus predominan en ambos miembros inferiores. Se puede ver clonus ocular horizontal. Los cambios en el estado mental comprenden agitación leve y verborrea. En el SS severo se observa hipertensión severa y taquicardia, que finalizan en un cuadro de shock. Estos pacientes se encuentran con delirio, agitados, con rigidez muscular e hipertonicidad. El aumento del tono es mayor en los miembros inferiores. La temperatura es mayor a $41{ }^{\circ} \mathrm{C}$, lo que pone en riesgo la vida (1-8). En el laboratorio puede hallarse rabdomiolisis, deterioro de la función renal, aumento de glóbulos blancos, aumento de transaminasas, y disminución del bicarbonato con acidosis metabólica (2-4). Como complicación se puede observar coagulación intravascular diseminada, leucopenia, trombocitopenia, arritmias, insuficiencia renal, síndrome de distrés respiratorio, convulsiones, coma y muerte (4). Se ha descrito como complicación rara de los ISRS, un síndrome de vasoconstricción cerebral reversible (13).

Los pacientes con manifestaciones leves pueden presentar sus síntomas de manera subaguda o crónica, mientras que en los casos graves pueden progresar rápidamente hacia a la muerte. La duración de los síntomas está dada en parte por la vida media de las drogas $(1,3)$. Usualmente $(70 \%$ de los casos), el SS resuelve con la discontinuación de la droga dentro de las 24 horas; del resto, el $40 \%$ requiere internación en terapia intensiva, y el $25 \%$ requiere intubación endotraqueal (4). La mortalidad se ve entre el 2 al $12 \%$ de los casos (3).

Coincidiendo con algunas series, todos nuestros pacientes fueron añosos y se presentaron con la tríada clásica de manifestaciones mentales, autonómicas y neuromusculares. El primer caso, una paciente en tratamiento con fluoxetina a la que se agregó omeprazol, con la aparición del síndrome a los cuatro días de la interacción. En los pacientes en tratamiento con ISRS habría que optar por ranitidina. El segundo caso es una paciente en tratamiento con mianserina, a la que se agregó escitalopram, con manejo no prescripto e inidóneo de la dosificación y la aparición del síndrome a los siete días. La tercera paciente se encontraba en tratamiento con ácido valproico y sertralina, tras el aumento inapropiado de esta última, comenzó con los síntomas en 24 horas. El último caso estaba en tratamiento con fluoxetina y amitriptilina, con la duplicación de esta última los síntomas aparecie- 
ron dentro de las 24 horas. Cabe resaltar que el paciente presentó hipertransaminasemia, anomalía de laboratorio bien descripta en el SS.

Dentro de los principales diagnósticos diferenciales deben considerarse la intoxicación por anticolinérgicos, que suele ser de inicio repentino ( $<12$ horas), caracterizada por reflejos y tono muscular normales, mucosas secas, piel eritematosa, caliente y seca, retención urinaria, ausencia de ruidos gastrointestinales, midriasis, excitación, delirio, hipertensión, taquicardia, taquipnea e hipertermia. Los ruidos hidroaéreos aumentados, hiperreflexia, sudoración, y el color de piel normal, distinguen al SS de la intoxicación por anticolinérgicos. La hipertermia maligna es una alteración farmacogenética caracterizada por hipertonicidad marcada, hiporreflexia y acidosis metabólica. La piel puede verse moteada con áreas cianóticas que contrastan con áreas rojizas. También presenta hipertensión, taquicardia, taquipnea e hipertermia. Habitualmente ocurre de minutos a horas luego de la exposición a agentes anestésicos inhalatorios. La rigidez extrema y la hiporreflexia la distinguen del SS. Por último, el síndrome neuroléptico maligno se caracteriza por su inicio lento, bradiquinesia o aquinesia, rigidez, fluctuaciones en el nivel de conciencia e inestabilidad autonómica, piel pálida y sudorosa, sialorrea, pupilas y ruidos gastrointestinales normales. También presenta hipertensión, taquicardia, taquipnea e hipertermia. El SS presenta hiperquinesia de comienzo súbito, lo que lo diferencia del síndrome neuroléptico (1-4). Otros diagnósticos a tener en cuenta son la sepsis, la encefalopatía herpética, el delirium tremens, el golpe de calor, la tormenta tiroidea, la intoxicación por salicilatos, el infarto agudo de miocardio, entidades que se presentan con hipertermia, o en forma de paroxismos o crisis, y la intoxicación con agentes adrenérgicos $(2-4,14)$. Siempre debería realizarse una punción lumbar para descartar una encefalitis, que podría presentarse de manera similar.

La evolución de este cuadro puede ser fatal, pero en la mayoría de los casos tiene buen pronóstico con la interrupción inmediata de la droga. La intensidad del tratamiento depende de la gravedad del cuadro (2). En los casos leves se utilizan medidas de soporte, suspensión del agente precipitante y tratamiento con benzodiacepinas. Los casos moderados, que presentan alteraciones cardiorrespiratorias y de temperatura, se deben tratar agresivamente; y para ellos podrían tener algún beneficio los antagonistas $5-\mathrm{HT}_{2 \mathrm{~A}}$. Estos pacientes generalmente requieren internación e infusión de soluciones electrolíticas, con el objetivo de mantener una diuresis por encima de $50-100 \mathrm{ml} / \mathrm{h}$, para evitar el daño por mioglobina a nivel renal. Se utilizan las benzodiacepinas para el tratamiento de las mioclonías, agitación y riesgo de convulsiones (1-4). En casos severos, con hipertermia mayor a $41,1^{\circ} \mathrm{C}$, se utiliza sedación, ventilación mecánica, parálisis neuromuscular. Para esto último se debe evitar la succinilcolina, por el mayor riesgo de arritmias en pacientes con hipercalemia por rabdomiolisis (2). No existe un rol demostrado de los agentes antipiréticos en el manejo del SS, ya que el incremento de la temperatura corporal se debe a la actividad muscular, y no a una alteración del centro hipotalámico de la temperatura (2).
El control de la inestabilidad autonómica involucra el control de la frecuencia del pulso y la presión arterial. Los cuadros de hipotensión suelen ser secundarios a la ingesta de IMAOs, y ésta se controla con bajas dosis de aminas simpaticomiméticas, como la noradrenalina (2). Los pacientes con hipertensión y taquicardia, que resultan del tratamiento presor o de la intoxicación por sí misma, deberían ser tratados con agentes de corta acción como el nitroprusiato o el esmolol (2). El tratamiento con propranolol, bromocriptina y dantrolene no han sido recomendados. El propranolol es un antagonista de los receptores 5- $\mathrm{HT}_{1 \mathrm{~A}}$ de larga duración, por lo que puede producir hipotensión y shock en pacientes con inestabilidad autonómica (2). La bromocriptina es un antagonista dopaminérgico, y ha sido implicada en el desarrollo del SS (2).

A pesar de que su eficacia no ha sido demostrada en estudios controlados, se ha propuesto la utilidad de la ciproheptadina, la clorpromazina, y la ziprasidona, por su acción bloqueante 5-HT, para el tratamiento del síndrome $(1,2,4)$. El tratamiento con ciproheptadina $\left(\right.$ Ciprovit $\left.^{\circledR}\right)$ requiere dosis de 12 a $32 \mathrm{mg}$ durante un período de $24 \mathrm{hs}$. Se debe considerar una dosis inicial de $12 \mathrm{mg}$ seguida de $2 \mathrm{mg}$ cada $2 \mathrm{hs}$, si los síntomas continúan. Una vez que el paciente se encuentra estable quedará con una dosis de $8 \mathrm{mg}$ cada 6 horas (2). La única presentación es para administración oral, y se puede utilizar por sonda nasogástrica. Produce sedación, y éste no debe ser un impedimento para el aumento de la dosis (2). Los antipsicóticos atípicos con antagonismo 5- $\mathrm{HT}_{2 \mathrm{~A}}$, como la olanzapina $10 \mathrm{mg}$, podría ser útil, pero su eficacia todavía no ha sido demostrada $(2,4)$. La clorpromazina $\left(\right.$ Ampliactil $\left.{ }^{\circledast}\right)$ es un antagonista de los receptores $5-\mathrm{HT}_{1 \mathrm{~A}} \mathrm{Y} 5-\mathrm{HT}_{2 \mathrm{~A}}$. Se recomienda el uso de $50 \mathrm{mg}$ por vía intramuscular en los casos leves, y 100 mg en los severos. Cuando se utiliza por vía oral, en los casos leves, la dosis es de $12,5 \mathrm{mg}$ cada 12 horas, y, en los casos moderados, de $25 \mathrm{mg}$ cada 12 horas $(2,4)$. Se ha asociado con hipotensión ortostática severa, distonías, síndrome neuroléptico maligno. A pesar de ello, los pacientes que requieren tratamiento endovenoso se encuentran internados, y a menudo están hipertensos; por lo que la hipotensión ortostática no suele representar un problema mayor. La hipotensión es la única contraindicación para su empleo (2). La ziprasidona (Zeldox ${ }^{\circledR}$ ) es un antipsicótico relativamente nuevo y el más potente inhibidor de los receptores $5-\mathrm{HT}_{1 \mathrm{~A}}$. Existe muy poca experiencia con esta droga en el SS. Un metanálisis de estudios no controlados compara el tratamiento con clorpromazina versus ciproheptadina; y demuestra mayor eficacia en morbimortalidad con la clorpromazina (15).

Esta entidad es habitualmente subdiagnosticada. Resulta fundamental tenerla presente y considerarla en todo paciente tratado con agentes serotoninérgicos, no sólo para hacer un diagnóstico temprano, sino para prevenir su aparición.

Educar a los pacientes para evitar la automedicación, y la restricción de combinaciones de fármacos que puedan interactuar y multiplicar la actividad de la transmisión serotoninérgica en el sistema nervioso resultan los pilares de la prevención. 


\section{Bibliografía}

1. Birmes P, Coppin D, Schmitt L, Lauque D. Serotonin syndrome: a brief review. CMAJ 2003; 168: 1439-42.

2. Boyer EW, Shannon M. The serotonin syndrome. N Engl J Med 2005; 352: 1112-20.

3. Mason PJ, Morris VA, Balcezak TJ. Serotonin syndrome. Presentation of 2 cases and review of the literature. Medicine (Baltimore) 2000; 79: 201-9.

4. Ener RA, Meglathery SB, Van Decker WA, Gallagher RM. Serotonin syndrome and other serotoninergic disorders. Am Acad of Pain Med 2003; 4: 63-74

5. Oates JA, Sjoerdsma A. Neurologic effects of tryptophan in patients receiving monoamine oxidase inhibitor. Neurology 1960; 10: 1076-8.

6. Insel TR, Roy BF, Cohen RM, Murphy DL. Possible development of the serotonin syndrome in man. Am J Psychiatry 1982; 139: 954-5.

7. Sternbach H. The serotonin syndrome. Am J Psychiatry 1991; 148: 70513 .

8. Dunkley EJ, Isbister GK, Sibbritt D, et al. The Hunter Serotonin Toxicity Criteria: Simple and accurate diagnostic decision rules for serotonin toxicity. QJM 2003; 96: 635-42.
9. Watson WA, Litovitz TL, Rodgers GC jr, et al. 2002 Annual report of the American Association of Poison Control Centers Toxic Exposure Surveillance System. Am J Emerg Med 2003; 21: 353-421.

10. Hemeryck A, Belpaire FM. Selective serotonin reuptake inhibitors and cytochrome P-450 mediated drug-drug interactions: An update. Curr Drug Metab 2002; 3: 13-37.

11. Liu ZQ, Zhu B, Tan YF, et al. O-dealkylation of fluoxetine in relation to CYP2C19 gene dose and involvement of CYP3A4 in human liver microsomes. JPET 2001; 299: 105-11.

12. Radomski JW, Dursum SM, Revely MA, Kutcher SP. An exploratory approach to the serotonin syndrome: An update of clinical phenomenology and revised diagnostic criteria. Med Hypotheses 2000; 55: 218-24.

13. Calabrese LH, Dodick DW, Schwedt TJ, Singhal AB. Reversible cerebral vasoconstriction syndromes. Narrative review. Ann Intern Med 2007; 146: 134-44.

14. Young P, Finn BC, Pellegrini D, et al. Paroxysmal sympathetic storm after diffuse axonal head injury. Medicina (B Aires) 2006; 66: 439-42.

15. Gillman PK. The serotonin syndrome and its treatment. J Psychopharmacol 1999; 13: 100-9. 